\title{
StABI5 Involved in the Regulation of Chloroplast Development and Photosynthesis in Potato
}

\author{
Tingting Zhu ${ }^{1,2,+}$, Linxuan Li ${ }^{1,+}$, Li Feng ${ }^{1,3}$ and Maozhi Ren 1,3,* \\ 1 Institute of Urban Agriculture, Chinese Academy of Agricultural Sciences, Chengdu 610213, China; \\ 20172602005t@cqu.edu.cn (T.Z.); lilinxuan@caas.cn (L.L.); 18202321576@163.com (L.F.) \\ 2 School of Life Sciences, Chongqing University, Chongqing 400045, China \\ 3 Zhengzhou Research Base, State Key Laboratory of Cotton Biology, Zhengzhou University, \\ Zhengzhou 450000, China \\ * Correspondence: renmaozhi01@caas.cn \\ + These authors have contributed equally to this work.
}

Received: 25 December 2019; Accepted: 4 February 2020; Published: 6 February 2020

\begin{abstract}
Abscisic acid (ABA) insensitive 5 (ABI5) - a core transcription factor of the ABA signaling pathway-is a basic leucine zipper transcription factor that plays a key role in the regulation of seed germination and early seedling growth. ABI5 interacts with other phytohormone signals to regulate plant growth and development, and stress responses in Arabidopsis, but little is known about the functions of ABI5 in potatoes. Here, we find that StABI5 is involved in the regulation of chloroplast development and photosynthesis. Genetic analysis indicates that StABI5 overexpression transgenic potato lines accelerate dark-induced leaf yellowing and senescence. The chlorophyll contents of overexpressed StABI5 transgenic potato lines were significantly decreased in comparison to those of wild-type Desiree potatoes under dark conditions. Additionally, the RNA-sequencing (RNA-seq) analysis shows that many metabolic processes are changed in overexpressed StABI5 transgenic potatoes. Most of the genes involved in photosynthesis and carbon fixation are significantly down-regulated, especially the chlorophyll $\mathrm{a}-\mathrm{b}$ binding protein, photosystem $\mathrm{I}$, and photosystem II. These observations indicate that StABI5 negatively regulates chloroplast development and photosynthesis, and provides some insights into the functions of StABI5 in regard to potato growth.
\end{abstract}

Keywords: chloroplast development; chlorophyll; photosynthesis; potato; StABI5

\section{Introduction}

Abscisic acid (ABA) is a pivotal phytohormone that regulates plant growth and development as well as abiotic and biotic stress responses [1-3]. ABA insensitive 5 (ABI5), which is a basic leucine zipper (bZIP) transcription factor, plays important roles in core ABA signaling by controlling seed dormancy, germination, plant growth, and stress responses. The abi5 mutant was originally obtained by screening ABA-insensitive phenotype from an Arabidopsis T-DNA mutant library [4]. The abi5 mutant has pleiotropic defects in ABA response, including decreased sensitivity to ABA-induced inhibition of germination and the altered expression of ABA-regulated genes [5,6], which suggests the role of $\mathrm{ABI} 5$ in abiotic stress response. ABI5 directly binds to the ABA-responsive element (ABRE) within the promoter regions of target genes containing early methionine-labeled 1 (EM1) and EM6 to regulate their expression. In the abi5 mutant, the expression levels of EM1 and EM6 have been shown to be significantly down-regulated, and displayed a phenotype that is insensitive to $\mathrm{ABA}$ and $\mathrm{NaCl}[6]$. Furthermore, ABI5 has an important function in seed germination by regulating the expression of polygalacturonase inhibiting protein 1 (PGIP1) and PGIP2 genes. ABI5 inhibits polygalacturonase activity by inducing the expression of PGIP1 and PGIP2 genes, which blocks seed coat rupture and 
inhibits seed germination [7]. In addition, recent studies show that ABI5 interacts with other plant hormone signals to regulate seed germination. Jasmonate-ZIM domain (JAZs), which are negative regulatory proteins of jasmonic acid (JA) signaling, repress the transcriptional activity of ABI5 and modulate seed germination in bread wheat and Arabidopsis [8]. The brassinosteroid insensitive 2 (BIN2) kinase, a key repressor of brassinosteroid (BR) signaling, has been shown to phosphorylate and stabilize ABI5 during seed germination [9]. By contrast, the brassinazole resistant 1 (BZR1) and BR insensitive 1-EMS-suppressor 1 (BES1) transcription factors of the BR signaling pathway can suppress ABI5 transcriptional activity and promote seed germination in Arabidopsis [10,11]. Similarly, DELLA proteins of gibberellic acid (GA) signaling promote the transcriptional activity of ABI5 to inhibit seed germination and postgerminative growth, whereas the transcription factor inducer of CBF expression 1 (ICE1) interferes with the transcriptional function of ABI5 and promotes seed germination [12,13].

In addition to seed germination, $\mathrm{ABI} 5$ also regulates postgerminative growth and vegetative tissue. ABI5 is not only expressed in root tips, stems and leaf veins, but also in the edges of old leaves and flowers. Diacylglycerol acyltransferase 1 (DGAT1), a rate-limiting enzyme of triacylglycerol biosynthesis, has been shown to be regulated by ABI5 to accumulate triacylglycerol in plant seedlings under stress [14]. Furthermore, ABI5 is also involved in regulating plant photosynthesis in Arabidopsis. For example, the staygreen 1 (SGR1) and non-yellow coloring 1 (NYC1) genes that are responsible for chlorophyll catabolism - containing an ABRE element in their promoters-are positively regulated by ABI5, reflecting that ABI5 is a negative regulator of photosynthesis through the activation of chlorophyll degradation [15]. The late embryogenesis abundant (LEA) protein ABA-response protein $(\mathrm{ABR})$ is regulated by ABI5 and is involved in dark-induced leaf senescence, implying that ABI5 plays a positive role in this process through the negative regulation of photosynthesis in Arabidopsis [16]. Besides, lateral root formation is also regulated by ABI5. ABA induces $A B I 5$ expression in the lateral root tips, and the effect of ABA- and nitrate-mediated lateral root growth inhibition is significantly reduced in abi4 and abi5 mutants [17].

The regulation of $A B I 5$ expression is complex and is mediated by multiple transcription factors and proteins. The ABI3 and ABI4 transcription factors of ABA signaling positively regulate the expression of $A B I 5$ during both seed germination and postgerminative growth $[18,19]$. Interestingly, ABI5 also directly binds to its own promoter through the G-box motif and activates the expression of itself [20]. The light-signaling transcription factor elongated hypocotyl 5 (HY5) binds to the promoter of $A B I 5$ gene and acts as a transcriptional activator of $A B I 5$ expression [21]. However, the B-box 21 (BBX21) is a negative regulator of $A B I 5$ expression-interfering with the binding of HY5 and ABI5 to the $A B I 5$ promoter [20]. Stress-activated transcription factor MYB96, a negative regulator of lateral root formation, activates the expression of $A B I 5$, which supports the role of ABI5 in the inhibition of lateral root growth $[17,22]$. By contrast, the other transcription factor MYB7 negatively regulates $A B I 5$ expression during seed germination [23]. Moreover, WRKY-domain transcription factors, such as WRKY18, WRKY40 and WRKY60, bind to the W-box motif within the promoter of ABI5 gene and thus repress $A B I 5$ expression during post-germinative growth [24]. The phosphorylation and stability of the ABI5 protein is regulated by its interaction with other proteins. In addition to SNF1-related protein kinases (SnRK2s), calcineurin B-like interacting protein kinase 26 (CIPK26), CIPK11 and BIN2 also phosphorylate ABI5 in vitro [9,25-27]. ABI5 dephosphorylation and destabilization depends on protein phosphate 2A (PP2A) and two catalytic subunits of PP6 phosphatase [28,29]. Additionally, ABI5 ubiquitination and 26S proteasomal degradation can be mediated by KEEP ON GOING (KEG) and CULLIN 4-based E3 ubiquitin ligases [30-32]. These studies show that ABI5 activity and stability are regulated by multiple posttranslational modifications.

However, despite the abundant evidence of the involvement of ABI5 in ABA and other signaling pathways during seed germination and post-germinative growth in Arabidopsis, details of the transcriptional regulation of StABI5 largely remain unknown in potato. In this study, the function of StABI5 in regulating chloroplast development and photosynthesis was identified. Genetic analysis indicated that StABI5 overexpression potato lines accelerated dark-induced leaf yellowing and senescence. Additionally, RNA-seq experiments were performed to test the functions of StABI5 in 
potato. The RNA-seq analysis showed that ribosomes, photosynthesis, and many metabolic processes were changed in StABI5 overexpression potatoes. Importantly, most chlorophyll a-b binding proteins and carbon fixation genes were down-regulated in StABI5 overexpression potatoes, implying that StABI5 is involved in the negative regulation of photosynthesis. Taken together, our results showed that StABI5 played important roles in the regulation of chloroplast development and photosynthesis in potato.

\section{Results}

\subsection{ABI5 Regulates Chlorophyll Catabolism Under Dark Conditions}

The transcription factor ABI5 not only plays an indispensable role in the ABA signaling pathway, but also plays a key role in seed maturation, germination, and early seedling growth regulation $[8,33]$. In addition, ABI5 also acts as an integrator for ABA and other plant hormone signals. Recent studies have shown that ABI5 is involved in photosynthesis by regulating chlorophyll metabolism [15,34,35]. To further analyze the function of ABI5 in chlorophyll metabolism, we treated the abi5-1 mutant and overexpressed AtABI5 transgenic Arabidopsis plants and leaves for 0, 5, and 8 days under dark conditions. The results showed that dark treatment can accelerate senescence and yellowing of leaves in Arabidopsis (Figure 1A,B). The abi5-1 mutant reduced leaf senescence and yellowing as compared to the wild type (WS), but overexpressed AtABI5 transgenic Arabidopsis accelerated this process. Furthermore, the chlorophyll content of the overexpressed AtABI5 transgenic lines was significantly lower than the Columbia (Col), whereas the abi5-1 mutant was higher than WS, which further verified the above results. To determine whether ABI5 promoted the expression of chlorophyll catabolism-related genes under dark conditions, we tested the transcription levels of chlorophyll catabolism-related genes $A t N Y C 1, A t C V$, and $A t P P H$ after dark treatment in Arabidopsis. These results showed that overexpressed AtABI5 transgenic Arabidopsis lines significantly upregulated the transcription levels of $A t N Y C 1, A t C V$, and AtPPH genes as compared to $\mathrm{Col}$ after dark treatment, while the abi5-1 mutant attenuated the up-regulation of these genes, which in turn delayed the senescence and yellowing of leaves (Figure 1C).
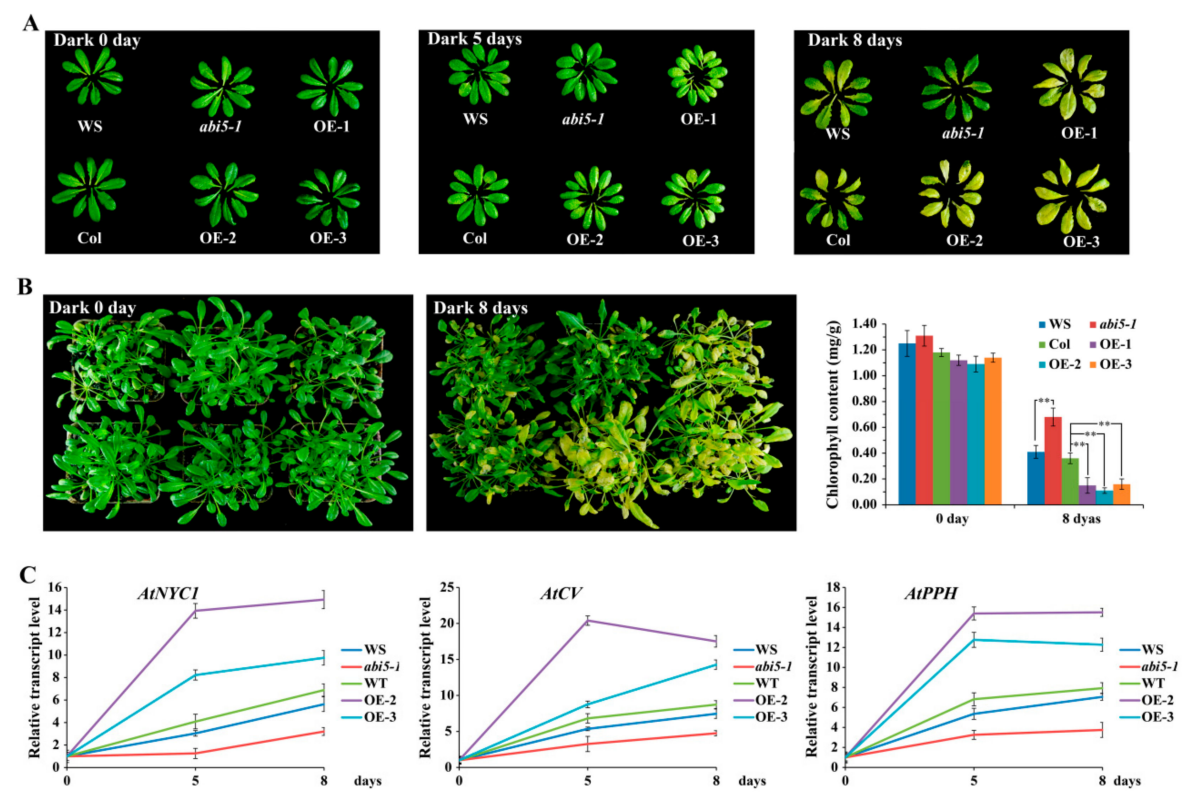

Figure 1. AtABI5 regulates chlorophyll catabolism in Arabidopsis. (A) Phenotype of the abi5-1 mutant and overexpressed AtABI5 (OE) transgenic Arabidopsis leaves after 0, 5, and 8 days of dark treatment, respectively. (B) Phenotype and chlorophyll content of the abi5-1 mutant and overexpressed AtABI5 (OE) transgenic lines after 0 and 8 days of dark treatment, respectively. The data represent the mean \pm $\mathrm{SD}$ of 3 independent experiments. Asterisks denote Student's t-test significant differences as compared with Col (** $P<0.01)$. (C) Transcription levels of chlorophyll catabolism-related genes in Arabidopsis after dark treatment. The data represents the mean \pm SD of 3 independent experiments. 


\subsection{Overexpressed StABI5 Potato Lines Accelerate Dark-Induced Leaf Yellowing and Senescence}

Potato (Solanum tuberosum) belongs to the Solanaceae family, whose tubers are edible and is the fourth largest food crop in the world after wheat, rice, and corn. In addition, it is also a kind of cash crop with significant advantages. Therefore, analyzing the roles of StABI5 in potato has important practical significance and economic value. A BLASTp analysis of the potato genome database (http://plants. ensembl.org/Solanum_tuberosum/Info/Index) was performed using the AtABI5 protein as reference. The BLASTp analysis results showed that the protein sequence of the PGSC0003DMG400002660 gene shared the highest similarity of $49.26 \%$ with AtABI5, so we named the gene as StABI5. The StABI5 gene sequence contains 4 exons and 3 introns, which encodes 428 amino acid residues with molecular mass of $46 \mathrm{kDa}$ (Figure S1A). Phylogenetic analysis of StABI5 and other species of ABI5 proteins showed that the evolutionary relationship between potato StABI5 and tomato SIABI5 is the closest, and it is the furthest from rice OsABI5 in regard to evolution (Figure S1B). Consistent with the Arabidopsis AtABI5 protein [33], StABI5 also contains conserved C1, C2, C3, bZIP, and C4 domains (Figure S1C).

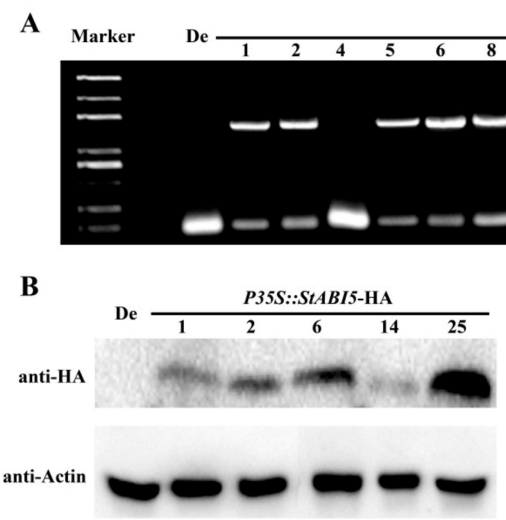

P35S::StABI5-HA
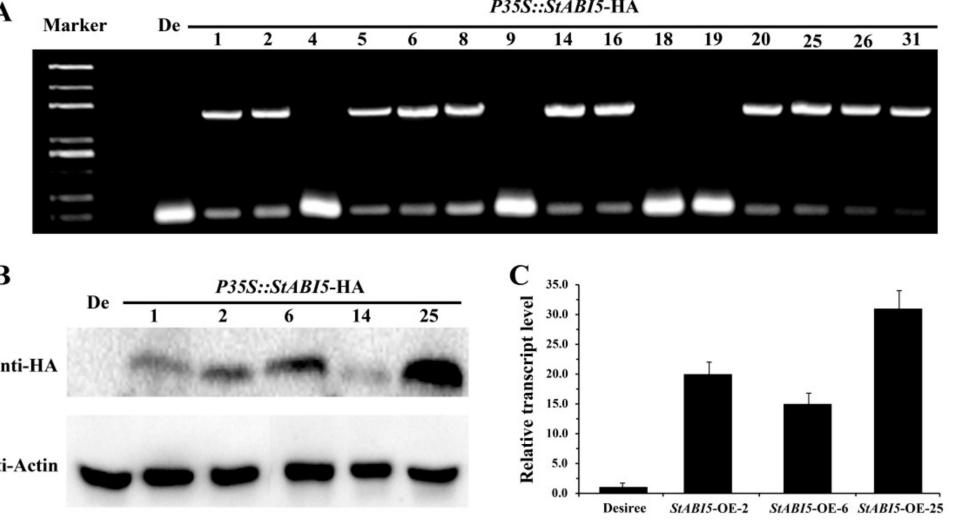

D

DIC
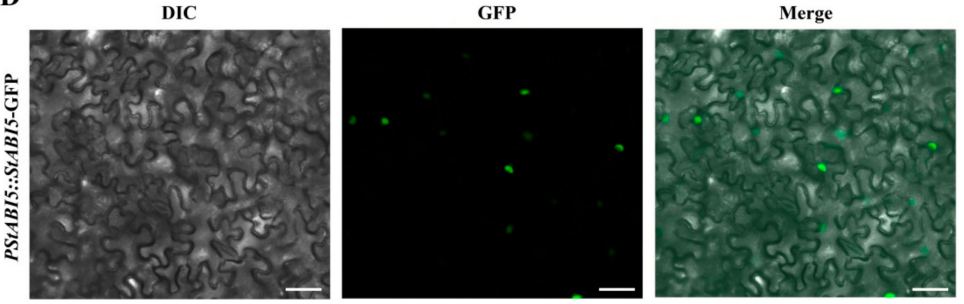

Figure 2. Identification and subcellular localization of StABI5 transgenic potatoes. (A) Identification of StABI5 transgenic potato lines by leaf PCR. The amplified PCR product was $1547 \mathrm{bp}$ when using primers P35S F and StABI5 R. De: Desiree. (B) Western blot analysis of StABI5 protein expression in StABI5 transgenic potato lines. (C) The transcription level of StABI5 in StABI5 transgenic potato lines obtained by qRT-PCR. StACTIN58 was used as an internal control. (D) Subcellular localization of StABI5 in tobacco (Nicotiana tabacum). Tobacco transiently expressed Agrobacterium strain containing the PStABI5::StABI5-GFP vector, and the localization of GFP was observed by laser confocal microscopy. Bars, $50 \mu \mathrm{m}$.

To further analyze the functions of StABI5 in potato, we constructed the StABI5 overexpression vectors P35S::StABI5-HA and PStABI5::StABI5-GFP. Through agrobacterium-mediated genetic transformation technology, we obtained transgenic potato lines with the P35S::StABI5-HA overexpression vector. We preliminarily identified transgenic lines containing the target vector by leaf PCR using primers P35S F and StABI5 R, and a total of 11 transgenic lines were identified (Figure 2A). Then, the protein expression of StABI5 in P35S::StABI5-HA transgenic potato lines was further detected by Western blot analysis. The results showed that the P35S::StABI5-HA-25 transgenic potato line had the highest protein expression level of StABI5, followed by the P35S::StABI5-HA-2 and P35S::StABI5-HA-6 lines (Figure 2B). Correspondingly, the StABI5 transcription level of the P35S::StABI5-HA-25 (StABI5-OE-25) line was also 
the highest among StABI5 transgenic potato lines (Figure 2C). To clarify the subcellular localization of StABI5 protein, we transiently expressed Agrobacterium strain containing the PStABI5::StABI5-GFP vector and observed the localization of GFP in tobacco. The results indicated that StABI5 was localized in the nucleus in tobacco (Figure 2D).

The overexpressed P35S::StABI5-HA (StABI5-OE) transgenic potato lines were planted in a field, and we found that StABI5-OE transgenic potato lines matured and aged significantly earlier than wild-type Desiree potatoes, and transgenic potato leaves showed phenotypes of yellowing and premature senescence (Figure S2), implying that StABI5 may participate in regulating chlorophyll metabolism. To verify whether StABI5 was involved in regulating chlorophyll metabolism, leaves of Desiree and StABI5-OE transgenic potato lines with similar phenotypes were treated with continuous darkness for 0, 2, 4 and 6 days, respectively. The results showed that StABI5-OE transgenic potato leaves accelerated yellowing and senescence under dark conditions (Figure 3A). Additionally, the chlorophyll contents of Desiree and StABI5-OE transgenic potato leaves were also measured. With an increase in darkness time, the chlorophyll contents of Desiree and StABI5-OE leaves were decreased, whereas StABI5-OE leaves showed a faster reduction in chlorophyll content (Figure 3B). These results indicated that StABI5 positively regulated chlorophyll catabolism under dark conditions in potato, which was consistent with the results in Arabidopsis.
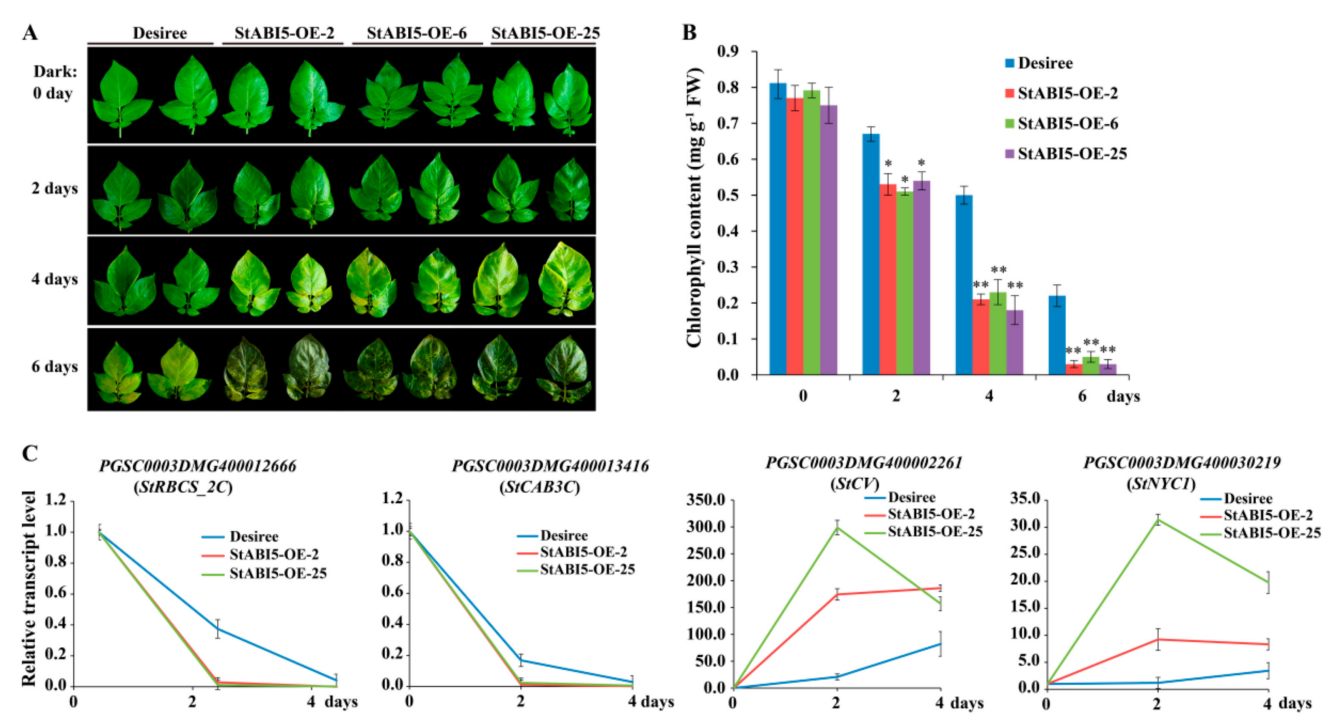

Figure 3. StABI5 regulates chlorophyll catabolism under dark conditions in potato. (A) Overexpressed StABI5 (StABI5-OE) transgenic potato leaves accelerate yellowing and senescence under dark conditions. (B) Chlorophyll contents of StABI5-OE transgenic potato and Desiree leaves under dark conditions. The data represent an average of five leaves with three duplicates. Error bars indicate \pm SD for triplicates. Asterisks denote Student's $t$-test significant differences as compared with Desiree potatoes $\left({ }^{*} P<0.05\right.$; $\left.{ }^{* *} P<0.01\right)$. (C) Transcription levels of chlorophyll metabolism-related genes PGSC0003DMG400012666 (StRBCS_2C), PGSC0003DMG400013416 (StCAB3C), PGSC0003DMG400002261 (StCV) and PGSC0003DMG400030219 (StNYC1) in StABI5-OE transgenic potato lines and Desiree leaves when treated with continuous darkness for 0,2 and 4 days.

To further test the effect of StABI5 on chlorophyll metabolism-related genes under dark conditions, we analyzed the transcription levels of chlorophyll metabolism-related genes by quantitative real-time PCR. Chlorophyll a/b-binding protein 3 (CAB3) is a member of chlorophyll a/b-binding protein family, which encodes the most abundant CAB mRNA in developing embryos and young leaves [36]. Rubisco small subunit (RBCS) is a key rate-limiting enzyme in plant photosynthesis [37]. The transcription levels of PGSC0003DMG400013416 (StCAB3C) and PGSC0003DMG400012666 (StRBCS_2C) genes were significantly down-regulated by dark treatment. Moreover, the transcription levels of StCAB3C and StRBCS_2C displayed the highest down-regulation rate in StABI5-OE lines as compared to that observed in wild-type Desiree potatoes when treated with continuous darkness for 2 days (Figure 3C). 
The AtCV and AtNYC1 genes positively regulate chloroplast degradation in Arabidopsis [15,38,39]. Consistent with the observations of $A t C V$ and AtNYC1 in Arabidopsis, the transcription levels of PGSC0003DMG400002261 (StCV) and PGSC0003DMG400030219 (StNYC1) genes were significantly up-regulated with dark treatment. However, the up-regulated rate of StCV and StNYC1 genes in StABI5-OE lines was significantly higher than that of Desiree potatoes (Figure 3C). Interestingly, StCV and StNYC1 genes contained conserved G-box element in their promoter regions, which implied that StABI5 binds to the promoters of the two genes to activate their expression. In addition, the expression levels of StCV and StNYC1 genes reached the highest level in StABI5-OE lines with dark treatment for 2 days, while the expression levels of the two genes were slowly increased during dark treatment for 0-4 days in Desiree potatoes. These results indicated that darkness can activate the transcriptional activity of StABI5 and accelerate chlorophyll degradation by inducing the expression of chlorophyll catabolism-related genes, which in turn causes leaf yellowing and senescence.

\subsection{Analysis of Differentially Expressed Genes (DEGs) Under Dark Conditions}

To further verify the functions of StABI5, we performed transcriptome sequencing using overexpressed StABI5 transgenic potato and wild-type Desiree leaves. According to previous experiments, we found that overexpressed StABI5 transgenic potato line 25 (StABI5-OE-25) had the highest protein expression level. Furthermore, the transcription levels of chlorophyll catabolism-related genes reached the highest in StABI5-OE-25 line when treated with darkness for 2 days. Therefore, we chose the StABI5-OE-25 line as the best experimental material and 2 days of continuous darkness as the best time node for transcriptome sequencing. RNA-seq was conducted in StABI5-OE-25 and Desiree leaves treated with continuous darkness for 2 days, respectively. After filtering the raw data, checking the sequencing error rate, and calculating the GC content distribution, we finally got clean reads for subsequent analysis (Table 1). Approximately $87 \%$ of the reads can be mapped to the annotated Solanum tuberosum genome, and more than $81 \%$ of the reads were uniquely mapped to the genome in each sample (Figure 4A). Here, 10,370 differentially expressed genes (DEGs) were found in StABI5 vs. De group, of which 5324 DEGs were up-regulated and 5046 DEGs were down-regulated. An amount of 8769 DEGs were found in StABI5_2DK vs. De_2Dk group, of which 4616 DEGs were up-regulated and 4153 DEGs were down-regulated (Figure 4B). The Venn diagram displayed that 4683 DEGs overlapped between StABI5 vs. De and StABI5_2DK vs. De_2Dk. Here, 13,594 DEGs overlapped between De_2Dk vs. De and StABI5_2DK vs. StABI5, and 3418 DEGs overlapped among the four groups (Figure 4C). Furthermore, hierarchical cluster analysis of DEGs was performed using the Cluster software package. The results showed that the expression levels of many genes were changed in StABI5-OE-25 line as compared to Desiree potatoes (Figure 4D).

Table 1. Summary of RNA-seq data.

\begin{tabular}{cccccccc}
\hline Sample & $\begin{array}{c}\text { Raw } \\
\text { Reads }\end{array}$ & $\begin{array}{c}\text { Clean } \\
\text { Reads }\end{array}$ & $\begin{array}{c}\text { Clean } \\
\text { Bases }\end{array}$ & $\begin{array}{c}\text { Error } \\
\text { Rate (\%) }\end{array}$ & $\begin{array}{c}\text { Q20 } \\
\mathbf{( \% )}\end{array}$ & $\begin{array}{c}\text { Q30 } \\
\mathbf{( \% )}\end{array}$ & $\begin{array}{c}\text { GC Content } \\
(\%)\end{array}$ \\
\hline De_1 & 51305060 & 49151244 & $7.37 G$ & 0.02 & 98.06 & 94.21 & 42.66 \\
De_2 & 49674828 & 47359662 & $7.1 \mathrm{G}$ & 0.02 & 98.21 & 94.57 & 42.83 \\
De_3 & 58613332 & 56616148 & $8.49 \mathrm{G}$ & 0.02 & 98.12 & 94.38 & 42.27 \\
StABI5_1 & 58077052 & 56123334 & $8.42 \mathrm{G}$ & 0.02 & 98.15 & 94.38 & 42.76 \\
StABI5_2 & 68409014 & 66871468 & $10.03 \mathrm{G}$ & 0.03 & 98.00 & 93.97 & 42.77 \\
StABI5_3 & 67555414 & 65226846 & $9.78 \mathrm{G}$ & 0.03 & 98.01 & 94.06 & 42.83 \\
De_2Dk_1 & 54602684 & 52122618 & $7.82 \mathrm{G}$ & 0.02 & 98.16 & 94.47 & 42.12 \\
De_2Dk_2 & 60997616 & 56806266 & $8.52 \mathrm{G}$ & 0.03 & 97.98 & 94.03 & 42.15 \\
De_2Dk_3 & 51539718 & 49560170 & $7.43 \mathrm{G}$ & 0.03 & 97.82 & 93.62 & 42.20 \\
StABI5_2DK_1 & 56909932 & 54989466 & $8.25 \mathrm{G}$ & 0.03 & 97.9 & 93.79 & 42.17 \\
StABI5_2DK_2 & 66680642 & 64385132 & $9.66 \mathrm{G}$ & 0.03 & 98.04 & 94.1 & 42.16 \\
StABI5_2DK_3 & 76207430 & 74339060 & $11.15 \mathrm{G}$ & 0.03 & 97.83 & 93.67 & 42.48 \\
\hline
\end{tabular}

De: Desiree; StABI5: StABI5-OE-25; De_2Dk: Desiree treated with continuous darkness for 2 days; StABI5_2DK: StABI5-OE-25 treated with continuous darkness for 2 days. 
A

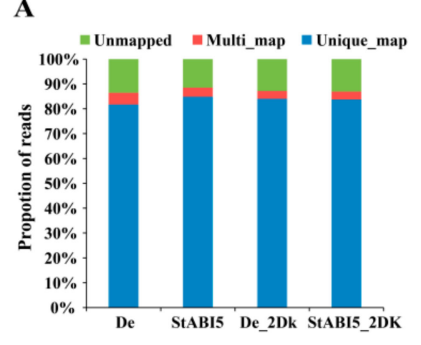

B

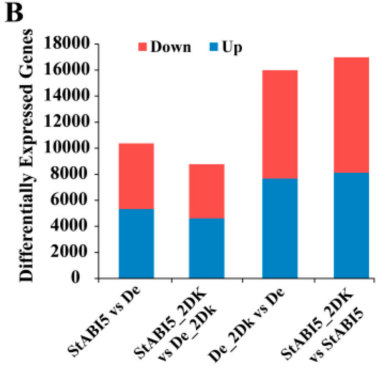

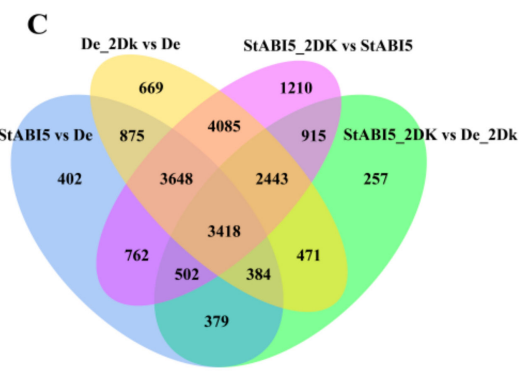

D

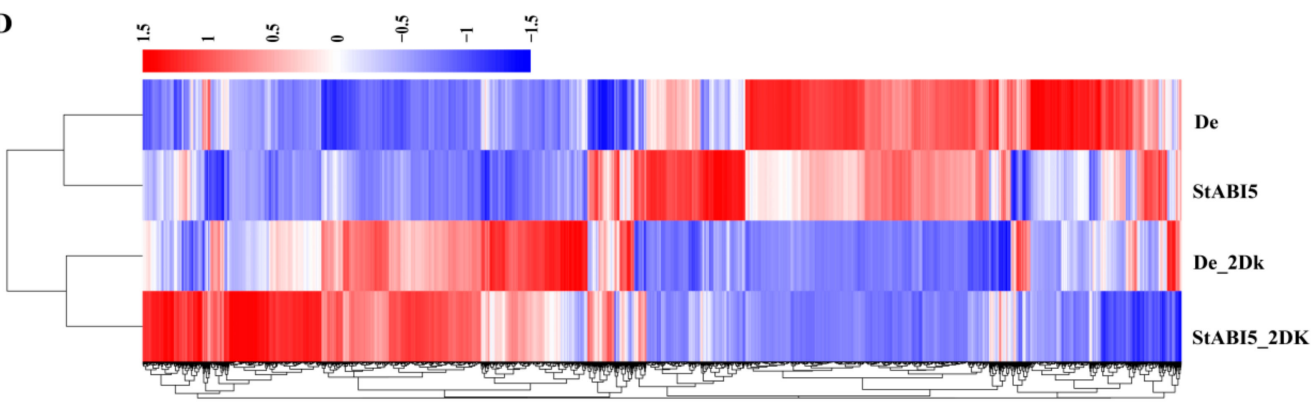

Figure 4. Analysis of differentially expressed genes. (A) Proportions of clean reads that were unmapped, mapped to multiple genes, and mapped to unique genes, which were plotted by three replicates of De, StABI5, De_2DK, and StABI5_2DK, respectively. (B) Statistical analysis of differentially expressed genes between different samples. (C) Analysis of the overlap of differentially expressed genes between different comparison combinations by Venn diagram. (D) Cluster analysis of differentially expressed genes by heat map. Red represents high gene abundance, blue represents low gene abundance.

\subsection{Gene ontology (GO) and KEGG Pathway Enrichment Analysis of DEGs}

To analyze the function of these DEGs and further understand the regulation of StABI5 in potato growth and development under dark conditions, we performed gene ontology (GO) assignments and enrichments. These DEGs were assigned to one or more of three categories: biological process, cellular component, and molecular function base on GO assignment, and they were significantly enriched in 153 and 113 terms of three GO categories in StABI5 vs. De and StABI5_2DK vs. De_2Dk groups, respectively (Supplementary Table S1). The most significant enrichment GO terms of the biological process were "photosynthesis" and "photosynthesis, light harvesting", of the cellular component were "thylakoid" and "chloroplast part", and those of the molecular function were "structural constituent of ribosome" and "chlorophyll binding" in StABI5 vs. De group (Figure 5A). Besides, the top three significantly enriched GO terms were "aromatic amino acid family metabolic process", "chloroplast part" and "carbon-carbon lyase activity" in StABI5_2DK vs. De_2Dk group (Figure 5B), suggesting that StABI5 regulates multiple cellular processes in potato.

To provide further insight into metabolic pathways and signal transduction pathways, KEGG pathway analysis of DEGs was performed. The top three enriched KEGG pathways were "ribosome", "photosynthesis-antenna proteins" and "photosynthesis" in StABI5 vs. De group (Figure 5C), of which "ribosome" and "photosynthesis" were the two most significantly down-regulated KEGG pathways, and "circadian rhythm-plant" was the most significantly up-regulated KEGG pathway. Additionally, the top three enriched KEGG pathways were "protein processing in endoplasmic reticulum", "carbon metabolism" and "phenylalanine metabolism" in StABI5_2DK vs. De_2Dk group (Figure 5D). Collectively, these results and observations suggested that StABI5 was involved in regulating various metabolic pathways in potato, especially photosynthesis. 
A

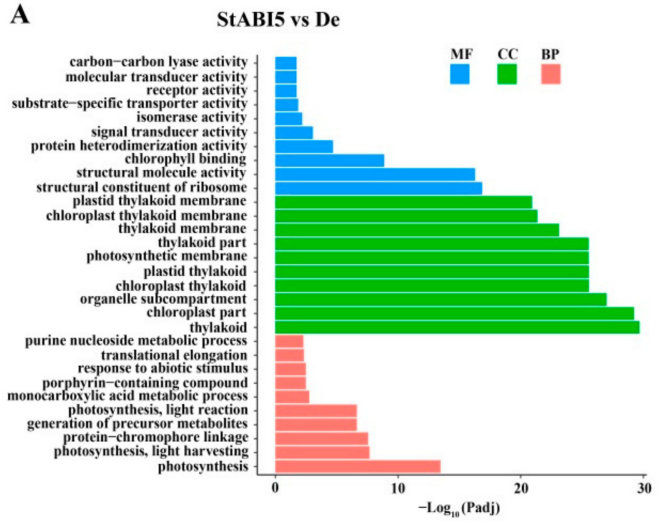

C

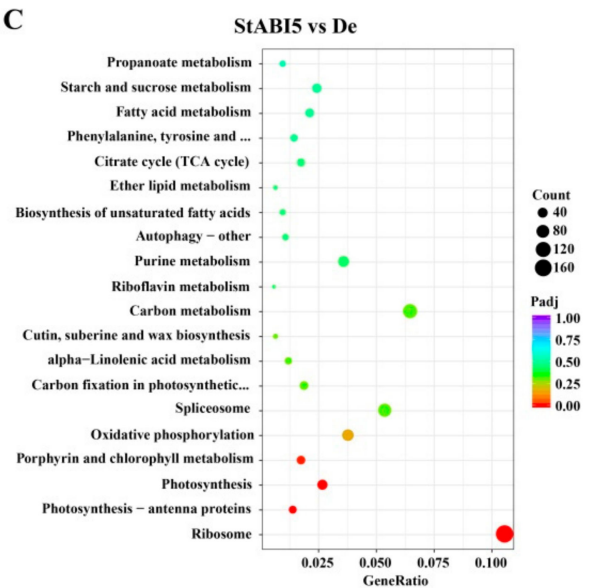

B

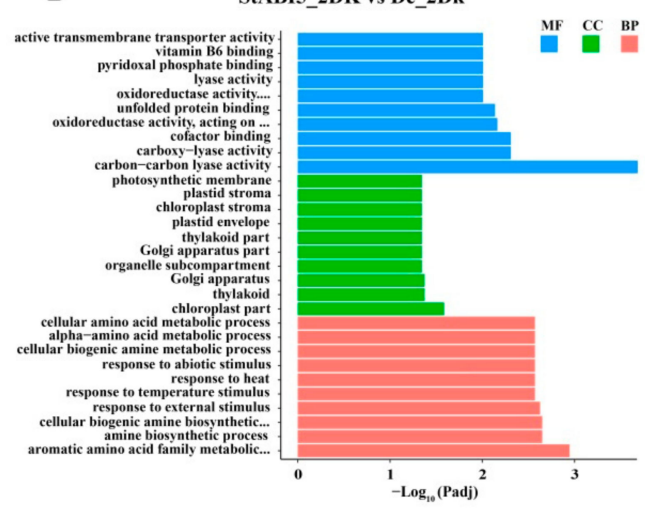

D

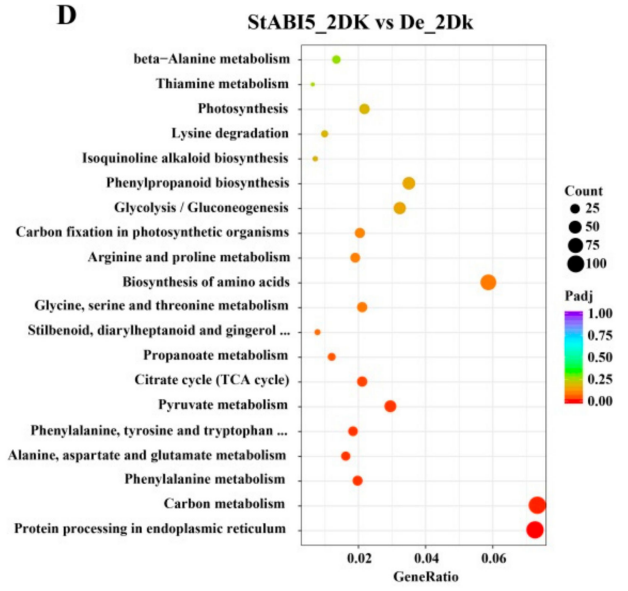

Figure 5. Gene ontology (GO) and KEGG pathway enrichment analysis of differentially expressed genes (DEGs). (A) The top 30 most enriched GO terms found in the analysis of DEGs in StABI5 vs. De group. Different colors represent molecular functions (MF), cellular components (CC), and biological processes (BP). Gene ontologies were ranked by their significance. (B) The top 30 most enriched GO terms found in the analysis of DEGs in StABI5_2DK vs. De_2Dk group. (C) The top 20 functionally enriched KEGG pathways found in the analysis of DEGs in StABI5 vs. De group. (D) The top 20 functionally enriched KEGG pathways found in the analysis of DEGs in StABI5_2DK vs. De_2Dk group.

\subsection{DEGs Involved in the Regulation of Chloroplast Development and Photosynthesis in Potato}

Chloroplasts contain the green pigment chlorophyll and are responsible for the light- driven reactions of photosynthesis, upon which essentially all life depends [40]. Chloroplast biogenesis and development plays a key role in photosynthesis efficiency. Previous studies have shown that ABI5 is involved in regulating plant photosynthesis in Arabidopsis. AtABI5 directly inhibits ABR gene expression, thereby suppressing photosynthesis and accelerating leaf senescence [16]. The promoters of chlorophyll catabolic genes SGR1 and NYC1 contain ABRE elements, and ABI5 activates the expression of these genes by binding to the ABRE elements to promote chlorophyll degradation [15]. The RNA-seq analysis showed that chlorophyll catabolic genes PGSC0003DMG400002261 (StCV) and PGSC0003DMG400016833 (StSGR1) were up-regulated 1.75- and 2.57-fold in StABI5 vs. De group, respectively. Furthermore, most genes involved in "photosynthesis" and "carbon fixation", such as chlorophyll a-b binding proteins, rubisco small subunits, and rubisco large subunits, were significantly down-regulated in StABI5 vs. De group (Table 2). Importantly, almost all genes of "chlorophyll a-b binding protein", "photosystem I" and "photosystem II" were down-regulated, indicating that StABI5 negatively regulated the expression of photosynthesis-related genes. Taken together, the RNA-seq data explain the phenotype of leaf yellowing in StABI5-OE lines. 
Table 2. StABI5 involved in the regulation of photosynthesis in potato.

\begin{tabular}{|c|c|c|c|}
\hline Gene_id & $\log 2 F C$ & Padj & Gene_Description \\
\hline \multicolumn{4}{|c|}{ Photosynthesis-antenna proteins } \\
\hline PGSC0003DMG400007787 & -1.91 & 5.51E-91 & Chlorophyll $\mathrm{a}-\mathrm{b}$ binding protein 8 \\
\hline PGSC0003DMG400006149 & -1.60 & 7.63E-91 & Chlorophyll $\mathrm{a}-\mathrm{b}$ binding protein 4 \\
\hline PGSC0003DMG400016695 & -1.83 & 4.07E-87 & Chlorophyll $\mathrm{a}-\mathrm{b}$ binding protein 50 \\
\hline PGSC0003DMG400004301 & -1.84 & $3.29 \mathrm{E}-58$ & Chlorophyll $\mathrm{a}, \mathrm{b}$ binding protein type I \\
\hline PGSC0003DMG400042498 & -1.64 & $7.88 \mathrm{E}-53$ & Chlorophyll $\mathrm{a} / \mathrm{b}$ binding protein \\
\hline PGSC0003DMG400013417 & -1.57 & 2.23E-51 & Chlorophyll a-b binding protein 3C \\
\hline PGSC0003DMG400033084 & -1.76 & $3.08 \mathrm{E}-48$ & Chlorophyll a/b-binding protein (cab-12) \\
\hline PGSC0003DMG400008804 & -1.47 & $2.13 \mathrm{E}-46$ & Chlorophyll $\mathrm{a} / \mathrm{b}$ binding protein \\
\hline PGSC0003DMG400026500 & -1.18 & $1.97 \mathrm{E}-44$ & Type I (26 kD) CP29 polypeptide \\
\hline PGSC0003DMG400023344 & -1.16 & $9.28 \mathrm{E}-43$ & Chlorophyll $\mathrm{a}-\mathrm{b}$ binding protein $6 \mathrm{~A}$ \\
\hline PGSC0003DMG400008298 & -1.51 & $1.38 \mathrm{E}-41$ & Chlorophyll $\mathrm{a} / \mathrm{b}$ binding protein \\
\hline PGSC0003DMG400012590 & -1.30 & $1.90 \mathrm{E}-40$ & Chlorophyll $\mathrm{a}-\mathrm{b}$ binding protein $\mathrm{CP} 24$ 10B \\
\hline PGSC0003DMG400004458 & -1.38 & $6.70 \mathrm{E}-38$ & Light-harvesting complex I protein Lhca5 \\
\hline PGSC0003DMG400019248 & -1.12 & $2.95 \mathrm{E}-36$ & Chlorophyll a-b binding protein 13 \\
\hline PGSC0003DMG400008564 & -1.09 & $3.60 \mathrm{E}-34$ & Chlorophyll $\mathrm{a}-\mathrm{b}$ binding protein 13 \\
\hline PGSC0003DMG400012591 & -1.08 & $6.93 \mathrm{E}-32$ & Chlorophyll a-b binding protein CP24 10A \\
\hline PGSC0003DMG400008488 & -0.90 & $4.29 \mathrm{E}-23$ & Chloroplast pigment-binding protein CP29 \\
\hline PGSC0003DMG400008309 & -2.19 & $6.76 \mathrm{E}-21$ & Chlorophyll $\mathrm{a} / \mathrm{b}$ binding protein \\
\hline PGSC0003DMG400021287 & -0.69 & $9.12 \mathrm{E}-17$ & Chlorophyll $\mathrm{a}-\mathrm{b}$ binding protein 8 \\
\hline PGSC0003DMG400002901 & -0.79 & $3.46 \mathrm{E}-16$ & Chlorophyll A/B binding protein \\
\hline PGSC0003DMG401009929 & 0.72 & $3.57 \mathrm{E}-12$ & Light-harvesting complex II protein Lhcb7 \\
\hline PGSC0003DMG400023461 & -0.48 & 5.67E-08 & Chlorophyll a-b binding protein $6 \mathrm{~A}$ \\
\hline \multicolumn{4}{|l|}{ Photosynthesis } \\
\hline PGSC0003DMG400004211 & -1.84 & $4.83 \mathrm{E}-88$ & Photosystem $\mathrm{Q}(\mathrm{B})$ protein \\
\hline PGSC0003DMG400005805 & -1.53 & $3.51 \mathrm{E}-67$ & Photosystem I reaction center subunit \\
\hline PGSC0003DMG400005890 & -1.55 & $1.25 \mathrm{E}-60$ & 16kDa membrane protein \\
\hline PGSC0003DMG400007536 & -1.37 & 8.99E-58 & Photosystem II reaction center W protein \\
\hline PGSC0003DMG400018434 & -1.33 & $1.57 \mathrm{E}-56$ & Oxygen evolving enhancer protein 3 \\
\hline PGSC0003DMG400010035 & -1.29 & $9.84 \mathrm{E}-56$ & Oxygen-evolving enhancer protein 1 \\
\hline PGSC0003DMG400020505 & -1.53 & $1.33 \mathrm{E}-48$ & Photosystem I reaction center subunit X \\
\hline PGSC0003DMG400000926 & -1.15 & $1.40 \mathrm{E}-47$ & - \\
\hline PGSC0003DMG400027672 & -1.32 & 4.17E-46 & Photosystem I subunit XI \\
\hline PGSC0003DMG400021727 & -1.15 & $7.78 \mathrm{E}-45$ & Photosystem II oxygen-evolving complex \\
\hline PGSC0003DMG400008585 & -1.24 & $9.79 \mathrm{E}-44$ & Photosystem II reaction center psb28 protein \\
\hline PGSC0003DMG400026667 & -1.23 & $7.16 \mathrm{E}-41$ & Isoform 2 of PsbP 2, chloroplastic \\
\hline PGSC0003DMG400002626 & -1.18 & $1.16 \mathrm{E}-40$ & Photosystem I psaH protein \\
\hline PGSC0003DMG400022022 & -1.09 & $6.81 \mathrm{E}-38$ & Photosystem I reaction center subunit IV B \\
\hline PGSC0003DMG400027671 & -1.11 & $2.60 \mathrm{E}-37$ & Photosystem I subunit XI \\
\hline PGSC0003DMG400020141 & -1.35 & $4.89 \mathrm{E}-35$ & Photosystem II reaction center $\mathrm{W}$ protein \\
\hline PGSC0003DMG400007201 & -1.04 & $3.06 \mathrm{E}-34$ & Photosystem II core complex proteins psbY \\
\hline PGSC0003DMG400046303 & -1.82 & $3.40 \mathrm{E}-34$ & Photosystem II CP47 chlorophyll apoprotein \\
\hline PGSC0003DMG400002782 & -0.90 & $2.78 \mathrm{E}-32$ & Oxygen-evolving enhancer protein 1 \\
\hline PGSC0003DMG400020466 & -1.01 & 1.17E-31 & ATP synthase subunit $b^{\prime}$, chloroplastic \\
\hline PGSC0003DMG400022249 & -1.05 & $6.05 \mathrm{E}-31$ & Chloroplast photosystem I reaction center V \\
\hline PGSC0003DMG400016504 & -1.02 & $2.05 \mathrm{E}-29$ & PSI-H \\
\hline PGSC0003DMG400016959 & -0.99 & $2.44 \mathrm{E}-29$ & ATP synthase delta chain, chloroplastic \\
\hline PGSC0003DMG400024531 & -1.01 & $5.13 \mathrm{E}-27$ & Thylakoid lumenal $25.6 \mathrm{kDa}$ protein \\
\hline PGSC0003DMG400035711 & -1.00 & $8.10 \mathrm{E}-27$ & Ferredoxin-1 \\
\hline PGSC0003DMG400007571 & -0.94 & $1.14 \mathrm{E}-26$ & PSI-H \\
\hline PGSC0003DMG400020484 & -0.82 & $8.96 \mathrm{E}-25$ & ATP synthase subunit $b^{\prime}$, chloroplastic \\
\hline PGSC0003DMG400014402 & -1.04 & $5.25 \mathrm{E}-22$ & Oxygen-evolving enhancer protein 3-1 \\
\hline PGSC0003DMG402003567 & -0.69 & $6.32 \mathrm{E}-20$ & Ferredoxin-NADP reductase \\
\hline PGSC0003DMG400021144 & -0.90 & 8.05E-20 & Photosystem I subunit III \\
\hline PGSC0003DMG400017556 & -0.76 & $2.01 \mathrm{E}-18$ & Photosystem II $22 \mathrm{kDa}$ protein, chloroplastic \\
\hline PGSC0003DMG400016482 & -0.68 & 3.14E-18 & ATP synthase gamma chain, chloroplastic \\
\hline PGSC0003DMG400018360 & -0.68 & $9.10 \mathrm{E}-14$ & Photosystem II $11 \mathrm{kDa}$ protein \\
\hline
\end{tabular}


Table 2. Cont.

\begin{tabular}{|c|c|c|c|}
\hline Gene_id & $\log 2 \mathrm{FC}$ & Padj & Gene_Description \\
\hline PGSC0003DMG400005372 & -1.92 & $4.12 \mathrm{E}-13$ & Photosystem I P700 chlorophyll a apoprotein \\
\hline PGSC0003DMG400011811 & 0.83 & $3.12 \mathrm{E}-10$ & Ferredoxin-NADP reductase \\
\hline PGSC0003DMG400011816 & -0.55 & $1.38 \mathrm{E}-09$ & Photosystem I reaction centre PSI-D subunit \\
\hline PGSC0003DMG402005881 & -0.95 & 5.17E-09 & Ferredoxin-3, chloroplast \\
\hline PGSC0003DMG400017532 & -0.55 & $2.16 \mathrm{E}-08$ & Ferredoxin-2 \\
\hline PGSC0003DMG400026360 & 1.41 & 8.72E-05 & Ferredoxin-3, chloroplast \\
\hline PGSC0003DMG400010353 & -0.66 & 0.000120 & Cytochrome c6, chloroplastic \\
\hline PGSC0003DMG400025106 & -1.39 & 0.000357 & ATP synthase epsilon chain, chloroplastic \\
\hline PGSC0003DMG400023985 & 1.80 & 0.003149 & Ferredoxin-3, chloroplast \\
\hline PGSC0003DMG400002312 & 2.55 & 0.028061 & Photosystem II 10 kDa polypeptide \\
\hline \multicolumn{4}{|c|}{ Carbon fixation in photosynthetic organisms } \\
\hline PGSC0003DMG400033037 & -2.38 & $4.99 \mathrm{E}-82$ & Ribulose bisphosphate carboxylase large chain \\
\hline PGSC0003DMG400012666 & -1.66 & $1.70 \mathrm{E}-67$ & Ribulose bisphosphate carboxylase small chain $2 \mathrm{C}$ \\
\hline PGSC0003DMG400019189 & -1.56 & $5.21 \mathrm{E}-39$ & Fructose-1,6-bisphosphatase \\
\hline PGSC0003DMG400019584 & -1.00 & $2.18 \mathrm{E}-33$ & Ribulose bisphosphate carboxylase small chain 1 \\
\hline PGSC0003DMG400007466 & 1.41 & 2.03E-23 & Phosphoenolpyruvate carboxylase \\
\hline PGSC0003DMG400004436 & -0.91 & 3.09E-21 & Triosephosphate isomerase, chloroplastic \\
\hline PGSC0003DMG400019188 & -0.79 & $1.42 \mathrm{E}-19$ & Chloroplast fructose-1,6-bisphosphatase I \\
\hline PGSC0003DMG400021264 & 0.87 & 7.73E-14 & Phosphoenolpyruvate carboxylase \\
\hline PGSC0003DMG400015385 & 0.75 & $2.84 \mathrm{E}-13$ & Phosphoenolpyruvate carboxylase \\
\hline PGSC0003DMG400029406 & -0.62 & $1.24 \mathrm{E}-12$ & Glyceraldehyde-3-phosphate dehydrogenase \\
\hline PGSC0003DMG400033874 & 0.58 & $1.92 \mathrm{E}-11$ & Malic enzyme \\
\hline PGSC0003DMG400003548 & -0.46 & $2.2 \mathrm{E}-10$ & Fructose-bisphosphate aldolase \\
\hline PGSC0003DMG400031063 & 0.47 & 1.38E-09 & Malate dehydrogenase \\
\hline PGSC0003DMG400011530 & -0.50 & $9.18 \mathrm{E}-09$ & Glyceraldehyde-3-phosphate dehydrogenase \\
\hline PGSC0003DMG400010788 & -0.55 & 4.54E-08 & Fructose-1,6-bisphosphatase, cytosolic \\
\hline PGSC0003DMG400020416 & 0.71 & $9.46 \mathrm{E}-08$ & Aspartate aminotransferase \\
\hline PGSC0003DMG400001595 & -0.43 & 2.99E-07 & Triosephosphate isomerase, chloroplastic \\
\hline PGSC0003DMG400010840 & 0.76 & 3.88E-07 & Aspartate aminotransferase \\
\hline PGSC0003DMG400011246 & 1.10 & $4.86 \mathrm{E}-07$ & Glyceraldehyde-3-phosphate dehydrogenase \\
\hline PGSC0003DMG400002675 & 0.58 & $9.16 \mathrm{E}-05$ & Fructose-bisphosphate aldolase \\
\hline PGSC0003DMG400016094 & 1.91 & 3.39E-03 & Phosphoenolpyruvate carboxykinase \\
\hline PGSC0003DMG400015637 & 0.45 & $1.82 \mathrm{E}-02$ & Aspartate aminotransferase \\
\hline PGSC0003DMG400011570 & 0.78 & 3.62E-02 & NAD-malate dehydrogenase \\
\hline
\end{tabular}

\subsection{StABI5 Involved in the Regulation of Plant Hormone Signal Transduction in Potato}

Plant hormones, including auxin, gibberellin (GA), cytokinin (CK), ABA, ethylene, and brassinosteroid, play important regulatory roles in plant growth and development, such as in seed germination, seedling growth, flowering, and fruit ripening. Recent studies indicated that ABI5 is involved in or regulated by these plant hormone signals [33,41,42]. Through the transcriptome data, we found that StABI5 was also involved in the regulation of plant hormone signaling in potato (Figure 6). Most genes related to the auxin pathway such as SAUR family protein and AUX were significantly changed, suggesting that StABI5 plays a role in the regulation of auxin signaling pathway (Table 3). In addition, the mRNA levels of genes associated with ABA, jasmonic acid, salicylic acid and brassinosteroid signals were up-regulated in StABI5 vs. De group. Importantly, all genes of $P Y R / P Y L$, $S n R K 2$, and $A B F$ were up-regulated, indicating that ABA pathway was activated in StABI5-OE-25 potato. Interestingly, we also found that most genes were up-regulated in the ethylene signaling pathway, especially EIN2/3 and ethylene response factor ERF1/2 genes, which were significantly up-regulated (Table 3), explaining the phenotype of leaves premature senescence in overexpressed StABI5 transgenic potato lines. These data showed that StABI5 was involved in the regulation of plant hormone signals to balance potato growth and development. 


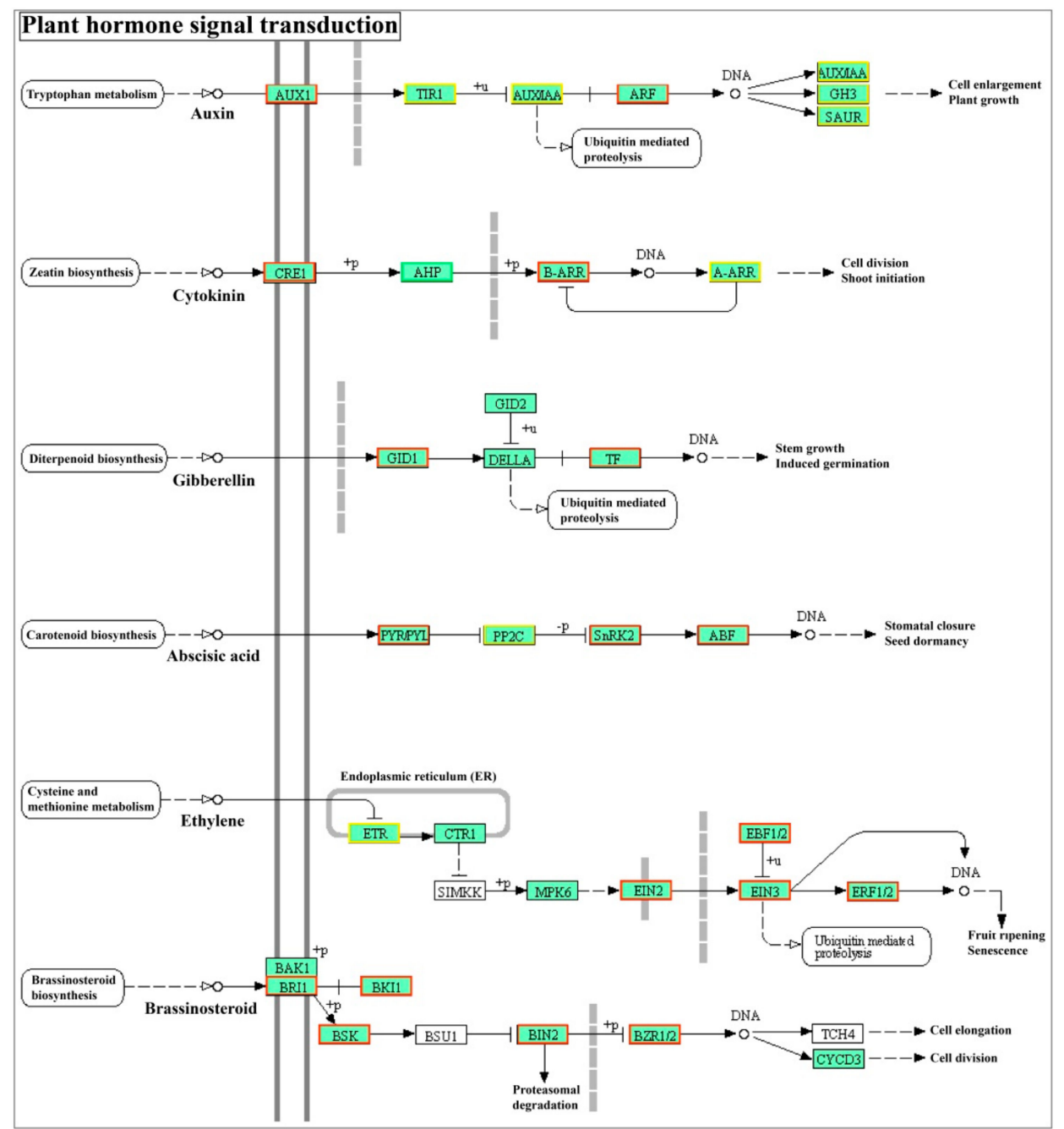

Figure 6. StABI5 involved in plant hormone signal transduction in potato. DEGs of plant hormone signals transduction were marked in StABI5 vs. De group. Green boxes represent down-regulated genes, red boxes indicate up-regulated genes, and yellow boxes represent both up- and down-regulated genes.

Table 3. DEGs of plant hormone signals transduction in StABI5 vs. De group.

\begin{tabular}{lccl}
\hline Gene_id & Log2FC & Padj & Gene_Description \\
\hline Auxin & & & \\
PGSC0003DMG400006550 & 0.39 & $4.77 \mathrm{E}-02$ & Auxin influx transport protein \\
PGSC0003DMG400024033 & 0.45 & $6.28 \mathrm{E}-04$ & Transport inhibitor response 1 \\
PGSC0003DMG400022404 & -0.38 & $3.84 \mathrm{E}-05$ & F-box family protein \\
PGSC0003DMG400019302 & -1.21 & $3.99 \mathrm{E}-04$ & Nt-iaa4.5 deduced protein \\
PGSC0003DMG400016317 & -0.39 & $7.77 \mathrm{E}-03$ & LEAUX= auxin-regulated protein homolog \\
PGSC0003DMG400002608 & 1.57 & $3.16 \mathrm{E}-10$ & ARF domain class transcription factor \\
PGSC0003DMG400002392 & 0.94 & $1.8 \mathrm{E}-20$ & Auxin response factor 7 \\
PGSC0003DMG400020711 & 0.84 & $1.5 \mathrm{E}-21$ & Auxin response factor 1 \\
PGSC0003DMG400031769 & 0.56 & $3.95 \mathrm{E}-08$ & Auxin response factor 3 \\
PGSC0003DMG400025856 & 1.64 & $7.91 \mathrm{E}-04$ & Auxin and ethylene responsive GH3 \\
PGSC0003DMG400024995 & -2.16 & $1.88 \mathrm{E}-04$ & Indole-3-acetic acid-amido synthetase GH3.6 \\
PGSC0003DMG400019274 & 3.10 & $1.32 \mathrm{E}-44$ & Indole-3-acetic acid-amido synthetase GH3.6 \\
PGSC0003DMG400024997 & 0.84 & $3.44 \mathrm{E}-14$ & Indole-3-acetic acid-amido synthetase GH3.6 \\
PGSC0003DMG400001605 & -2.07 & $3.18 \mathrm{E}-02$ & Conserved gene of unknown function \\
PGSC0003DMG400001614 & -1.26 & $1.81 \mathrm{E}-12$ & SAUR family protein \\
PGSC0003DMG400001668 & -1.26 & $9.96 \mathrm{E}-05$ & SAUR family protein \\
PGSC0003DMG400001667 & -0.68 & $5.17 \mathrm{E}-09$ & SAUR family protein \\
PGSC0003DMG400001601 & 2.54 & $2.23 \mathrm{E}-02$ & Auxin-responsive family protein \\
PGSC0003DMG400026709 & 1.15 & $1.10 \mathrm{E}-02$ & SAUR family protein \\
\hline
\end{tabular}


Table 3. Cont.

\begin{tabular}{|c|c|c|c|}
\hline Gene_id & $\log 2 \mathrm{FC}$ & Padj & Gene_Description \\
\hline \multicolumn{4}{|l|}{ Cytokinin } \\
\hline PGSC0003DMG400029463 & 0.31 & 2.90E-02 & Cytokinin receptor 1 \\
\hline PGSC0003DMG400003196 & 1.12 & $5.04 \mathrm{E}-12$ & Two-component system sensor histidine kinase/response regulator \\
\hline PGSC0003DMG400016643 & 2.32 & 1.33E-08 & Two-component system sensor histidine kinase/response regulator \\
\hline PGSC0003DMG400007823 & 0.42 & $3.71 \mathrm{E}-03$ & Two-component system sensor histidine kinase/response regulator \\
\hline PGSC0003DMG400027597 & -2.45 & $8.96 \mathrm{E}-03$ & Type-a response regulator \\
\hline PGSC0003DMG400029852 & -1.25 & 9.99E-08 & Type-a response regulator \\
\hline \multicolumn{4}{|l|}{ Gibberellin } \\
\hline PGSC0003DMG400021991 & 1.49 & $3.54 \mathrm{E}-18$ & GID1-like gibberellin receptor \\
\hline PGSC0003DMG400003849 & 1.33 & $5.66 \mathrm{E}-09$ & GID1-like gibberellin receptor \\
\hline PGSC0003DMG400015897 & 0.89 & 2.79E-05 & Abscisic acid receptor PYL4 \\
\hline PGSC0003DMG400012155 & 0.40 & $1.45 \mathrm{E}-03$ & Pathogenesis-induced protein \\
\hline PGSC0003DMG400029194 & 1.94 & $1.21 \mathrm{E}-02$ & Abscisic acid receptor PYL4 \\
\hline PGSC0003DMG400009112 & -0.96 & $3.52 \mathrm{E}-02$ & Protein phosphatase $2 \mathrm{C}$ \\
\hline PGSC0003DMG400002573 & 0.41 & 4.73E-05 & Protein phosphatase $2 \mathrm{C}$ ABI2 homolog \\
\hline PGSC0003DMG400016742 & 1.17 & 2.03E-03 & Protein phosphatase $2 \mathrm{C}$ AHG3 homolog \\
\hline PGSC0003DMG400029297 & 0.64 & $1.16 \mathrm{E}-08$ & Abscisic insensitive $1 \mathrm{~B}$ \\
\hline PGSC0003DMG400025895 & 1.07 & 2.62E-15 & Serine/threonine-protein kinase SAPK10 \\
\hline PGSC0003DMG400029774 & 0.63 & $1.31 \mathrm{E}-03$ & Basic-leucine zipper \\
\hline PGSC0003DMG400008011 & 0.38 & 2.27E-02 & ABRE binding factor \\
\hline PGSC0003DMG400002660 & 11.15 & $3.63 \mathrm{E}-63$ & DNA binding protein, ABI5 \\
\hline PGSC0003DMG400030928 & 0.55 & 5.97E-06 & EIN3-binding F-box protein 1 \\
\hline PGSC0003DMG400002914 & 1.05 & $1.41 \mathrm{E}-27$ & EIN3-binding F-box protein 1 \\
\hline PGSC0003DMG400008712 & 0.37 & $5.48 \mathrm{E}-03$ & EIL2 \\
\hline PGSC0003DMG400017231 & 1.49 & $1.09 \mathrm{E}-04$ & Transcription factor TSRF1 \\
\hline PGSC0003DMG400014204 & 1.85 & $2.26 \mathrm{E}-02$ & Transcription factor TSRF1 \\
\hline PGSC0003DMG400014594 & 2.01 & $9.78 \mathrm{E}-13$ & Ethylene response factor \\
\hline \multicolumn{4}{|l|}{ Brassinosteroid } \\
\hline PGSC0003DMG400019698 & 0.38 & 2.41E-04 & BRI1 protein \\
\hline PGSC0003DMG400015442 & 0.52 & $6.35 \mathrm{E}-05$ & Conserved gene of unknown function \\
\hline PGSC0003DMG400009972 & 1.08 & $1.74 \mathrm{E}-23$ & Receptor protein kinase \\
\hline PGSC0003DMG400001670 & 0.29 & $2.38 \mathrm{E}-03$ & Receptor protein kinase \\
\hline PGSC0003DMG400026744 & 0.80 & 7.13E-11 & Glycogen synthase kinase- 3 beta \\
\hline PGSC0003DMG400028428 & 0.55 & 8.79E-12 & Glycogen synthase kinase- 3 beta \\
\hline \multicolumn{4}{|l|}{ Jasmonic acid } \\
\hline PGSC0003DMG400032119 & 0.48 & $1.00 \mathrm{E}-05$ & Jasmonate ZIM-domain protein 3 \\
\hline PGSC0003DMG400002930 & 1.96 & $2.56 \mathrm{E}-21$ & Jasmonate ZIM-domain protein 1 \\
\hline PGSC0003DMG400022888 & 0.76 & 1.17E-09 & Salt responsive protein 1 \\
\hline PGSC0003DMG400029237 & 0.59 & $1.34 \mathrm{E}-05$ & Gene of unknown function \\
\hline PGSC0003DMG400015667 & 0.36 & 2.63E-02 & Pto-responsive gene 1 protein \\
\hline \multicolumn{4}{|l|}{ Salicylic acid } \\
\hline PGSC0003DMG401000923 & 1.79 & $1.02 \mathrm{E}-47$ & NIM1 2 \\
\hline
\end{tabular}

\subsection{Validation of Transcriptome Data by Quantitative Real-Time PCR}

To confirm these transcriptome-based observations and the reliability of RNA-seq data, fifteen differentially expressed genes were randomly selected from the RNA-seq data in StABI5 vs. De group for quantitative real-time PCR. The changes in the transcription levels of 15 genes, including 
PGSC0003DMG400002660 and PGSC0003DMG400001390, displayed the same trends as gained in RNA-seq data (Figure S3), indicating that the RNA-seq data were reliable and valid.

\section{Discussion}

Plants have evolved a set of precise molecular regulatory mechanisms in response to external biotic and abiotic stresses. ABA is one of the most important phytohormones to fight against environmental stresses in various plants $[1,2,43]$. It also has an essential role in multiple physiological processes of plants, such as seed germination, lateral root formation, leaf senescence, and stomatal closure [43,44]. In-depth dissection of the biological functions of essential regulatory proteins in the ABA signaling pathway is necessary for understanding physiological and metabolic processes involved in ABA signaling. The ABA signaling pathway is a complex signaling network that integrates with other signaling pathways including BR, CK, JA, GA and auxin signals. Many studies have shown that $\mathrm{ABI}$, as a key positive transcription factor in the $\mathrm{ABA}$ signaling pathway, not only responds to $\mathrm{ABA}$ to regulate the expression of ABA-responsive genes, but also participates in the regulation of other plant hormone signaling pathways [10,33,41,42]. Components of auxin, CK, GA, JA and BR signals were shown to take part in ABI5 regulation or to be regulated by ABI5 [10,33,41,42]. For example, ABI5 negatively regulates PIN1 accumulation and represses auxin activity in roots [45]. In this study, the transcriptome data results showed that mRNA levels of many genes involved in auxin, CK, GA, BR, ethylene and JA signaling pathways were changed in overexpressed StABI5 transgenic potatoes, suggesting that StABI5 is also involved in regulating these plant hormone signaling pathways in potato.

Photosynthesis is a series of physiological and biochemical processes in chloroplasts. The ability of photosynthesis determines plant growth status, while chloroplast biogenesis and function affect the efficiency of photosynthesis. Recent studies have shown that photosynthesis is regulated by the target of rapamycin (TOR) and ABA signaling pathways [42,46,47]. TOR signaling acts as a positive regulatory factor to promote plant chloroplast biogenesis and photosynthesis, whereas ABA signaling acts as a negative regulatory signal to inhibit photosynthesis [42,47-50]. The transcription factor ABI4 of the ABA signaling pathway binds to the promoter regions of photosynthetic genes such as $\angle H C B$ and $R B C S$, suppressing the expression of these genes and thus inhibiting photosynthesis [51,52]. Furthermore, ABI4 negatively regulates cotyledon greening and inhibits photosynthesis and seedling growth in Arabidopsis [50]. AtABI5 directly binds the promoter regions of chlorophyll catabolism genes AtSGR1 and AtNYC1 to activate the expression of the two genes in Arabidopsis [15,34,35], suggesting that AtABI5 negatively regulates photosynthesis by promoting chlorophyll degradation. Under dark conditions, AtABI5 inhibits the expression of $A B R$ gene, accelerating leaf senescence [16]. Therefore, these studies show that ABI5 acts as a negative regulator of photosynthesis in Arabidopsis. In this study, we further verified that StABI5 was involved in regulating chloroplast development and photosynthesis in potato. In the dark, overexpressed StABI5 transgenic potato leaves displayed accelerated yellowing and senescence phenotypes. The chlorophyll content between overexpressed StABI5 transgenic potato lines and Desiree potatoes displayed a significant difference under dark conditions. Besides, we found that chlorophyll catabolic genes StCV, StSGR1, and StNYC1 contained conserved G-box element in their promoter regions, and the mRNA levels of these genes were up-regulated in StABI5 transgenic potato lines, showing that darkness activates the transcriptional activity of StABI5, which degrades chlorophyll by inducing the expression of chlorophyll catabolism-related genes. However, whether StABI5 directly binds to promoter regions of $S t C V$, StSGR1, and StNYC1 genes still requires further study.

Through analysis of the RNA-seq data, we found that many intracellular metabolic processes were changed, such as photosynthesis and carbon metabolism. Importantly, most of genes involved in "photosynthesis" and "carbon fixation" were significantly down-regulated in StABI5 vs. De group. Chlorophyll a-b binding proteins are components of photosynthetic antenna complexes and play vital roles in photosystems I and II [53]. The disruption of chlorophyll a-b binding proteins reduces photosynthesis efficiency [53,54]. The RNA-seq data showed that all genes of chlorophyll a-b binding proteins were down-regulated in overexpressed StABI5 transgenic potatoes, indicating that StABI5 
negatively regulates the expression of photosynthesis-related genes. Collectively, these observations, together with the findings in the RNA-seq data, suggested that StABI5 negatively regulates chloroplast development and photosynthesis in potato.

\section{Materials and Methods}

\subsection{Plant Materials and Growth Conditions}

Wild type Arabidopsis Columbia (Col) and Wassileskija (WS) seeds were used in this study. The abi5-1 mutant seeds from the WS background were obtained from Dr. Zhizhong Gong. Wild-type Desiree potato seedlings were provided by Professor Jin Liping. The main overexpression vectors P35S::AtABI5-HA, P35S::StABI5-HA, and PStABI5::StABI5-GFP were used in this study. The Arabidopsis seeds were surface sterilized using liquid methods. The seeds were first treated with $70 \%$ ethanol for $2 \mathrm{~min}$ and then the supernatant was discarded. Then, the seeds were treated with $10 \%$ sodium hypochlorite, containing $0.3 \%$ Tween-20, for $5 \mathrm{~min}$, and the supernatant was discarded. This was followed by rinsing four times with sterile water, centrifugation at $4000 \times g$ for 2 min each time, and the supernatant was discarded. Finally, the seeds were suspended in $0.15 \%$ sterile agarose and kept at $4{ }^{\circ} \mathrm{C}$ for 2 days. Sterilized seeds were plated on plates and grown in a growth chamber at $22{ }^{\circ} \mathrm{C}$ under $16 \mathrm{~h}$ of $60-80 \mu \mathrm{E} \cdot \mathrm{m}^{-2} \mathrm{~s}^{-1}$ continuous light and $8 \mathrm{~h}$ of darkness.

\subsection{Dark Treatment of Overexpression AtABI5 Transgenic Arabidopsis}

The seeds of Col, WS, abi5-1 and overexpressed AtABI5 Arabidopsis seeds were sterilized and then placed on a $0.5 \times$ MS solid medium for 10 days in a growth chamber at $22{ }^{\circ} \mathrm{C}$ under $16 \mathrm{~h}$ of continuous light and $8 \mathrm{~h}$ of darkness. Then the seedlings were transplanted to small flowerpots for 4 weeks under normal conditions. Seedlings of Col, WS, abi5-1, and overexpressed AtABI5 Arabidopsis were transferred to dark conditions for 8 days.

\subsection{Dark Treatment of Arabidopsis and Potato Leaves}

Seedlings of Col, WS, abi5-1, and overexpressed AtABI5 Arabidopsis were transplanted to small flowerpots for 4 weeks under normal conditions, and then the leaves of Col, WS, abi5-1 and overexpressed AtABI5 Arabidopsis plants were placed in 6-well plates with sterile water for 8 days under dark conditions. Finally, the leaf phenotypes were observed.

Seedlings of Desiree and overexpressed StABI5 (StABI5-OE) transgenic potato lines were grown in flowerpots for approximately 4 weeks at $22{ }^{\circ} \mathrm{C}$ under $16 \mathrm{~h}$ of continuous light and $8 \mathrm{~h}$ of darkness. Then, the leaves of the Desiree and StABI5-OE plants were cultured for 6 days in continuous darkness in a wet filter paper tray and the phenotypes of the leaves were observed.

\subsection{Chlorophyll Content Measurement}

The chlorophyll content of the Arabidopsis and potato leaves was measured for 0-8 days under dark conditions. Chlorophyll was extracted from the plant leaves and then quantified [55]. This was done by weighing $0.5 \mathrm{~g}$ samples in $15 \mathrm{~mL}$ centrifuge tubes, placing the tubes into a $-20{ }^{\circ} \mathrm{C}$ refrigerator for $2 \mathrm{~h}$. Then the tubes were taken out and $10 \mathrm{~mL} 80 \%$ acetone was added. The tubes were stored at $50{ }^{\circ} \mathrm{C}$ for $4 \mathrm{~h}$ in a dark cabinet. After mixing, chlorophyll content was determined photometrically by measuring absorption at $663 \mathrm{~nm}$ and $645 \mathrm{~nm}$, and chlorophyll content was then calculated as described previously [16].

\subsection{Total RNA Extraction and Detection for Transcriptome Sequencing}

The leaves of the StABI5-OE-25 line and wild-type Desiree were treated with darkness for 0 and 2 days. Total RNA of the StABI5-OE-25 line and Desiree leaves, including those dark-treated 0 and 2 days, were isolated using the RNAprep Pure Plant Kit (TIANGEN, Beijing, China), respectively. For each treatment, three independent biological replicates were performed. RNA purity was checked 
using a NanoPhotometer ${ }^{\circledR}$ spectrophotometer. RNA concentration was measured using Qubit ${ }^{\circledR}$ RNA Assay Kit in Qubit ${ }^{\circledR}$ 2.0 Flurometer. RNA integrity was assessed using the RNA Nano 6000 Assay Kit with the Bioanalyzer 2100 system.

\subsection{RNA library Construction and Transcriptome Sequencing}

A total amount of $3 \mu \mathrm{g}$ RNA per sample was used as input material for the RNA sample preparations. Sequencing libraries were generated using NEBNext ${ }^{\circledR}$ UltraTM RNA Library Prep Kit for Illumina ${ }^{\circledR}$ (NEB, Ipswich, MA, USA) following the manufacturer's recommendations and index codes were added to attribute sequences to each sample. After cluster generation, the library preparations were sequenced on an Illumina platform and $150 \mathrm{bp}$ paired-end reads were generated.

\subsection{Reads Mapping to the Reference Genome}

Clean data (clean reads) were obtained by removing adapter, ploy- $\mathrm{N}$, and low quality reads from the raw data. At the same time, the Q20, Q30 and GC content of the clean data were calculated. All the downstream analyses were based on the clean data with high quality. Reference genome and gene model annotation files were downloaded from the potato genome database website (http: //plants.ensembl.org/Solanum_tuberosum/Info/Index). Index of the reference genome was built using Hisat2 (v2.0.5) and paired-end clean reads were aligned to the reference genome using Hisat2 (v2.0.5) [56].

\subsection{Differential Expression Analysis}

A differential expression analysis of two groups was performed using the DESeq2 $\mathrm{R}$ package (1.16.1) [57]. The resulting P-values were adjusted using the Benjamini and Hochberg's approach for controlling the false discovery rate. Genes with an adjusted $P$-value (Padj) $<0.05$ found by DESeq2 were assigned as differentially expressed genes (DEGs).

\subsection{GO and KEGG Enrichment Analysis of Differentially Expressed Genes}

A gene ontology (GO) enrichment analysis of DEGs was implemented by the clusterProfiler $\mathrm{R}$ package, where gene length bias was corrected. GO terms with corrected $\mathrm{P}$-value $<0.05$ were considered significantly enriched by differentially expressed genes. KEGG is a database resource for understanding high-level functions and utilities of biological systems, such as cells, organisms and ecosystems, from molecular level information, especially large-scale molecular datasets generated by genome sequencing and other high-through put experimental technologies. We used the clusterProfiler $\mathrm{R}$ package (with a corrected $P$-value $<0.05$ ) to test the statistical enrichment of differentially expression genes in KEGG pathways.

\subsection{Quantitative Real-Time PCR}

Total RNA of both the StABI5-OE transgenic potato and Desiree leaves, including those treated with darkness for 0 and 2 days, was isolated using the RNAprep Pure Plant Kit (TIANGEN, Beijing, China). The PrimeScript ${ }^{\circledR}$ RT reagent kit (Takara, Dalian, China) was used for reverse transcription following the manufacturer's instructions. Relative transcription levels were assayed by two-step real-time PCR analysis using the CFX96 real-time PCR system (BIO-RAD, USA). Real-time primers were designed by Primer premier 5.0 and the details were presented in Supplementary Table S2. StACTIN58 was used as an internal control. The data represent the mean \pm SD of three independent experiments.

Supplementary Materials: Supplementary materials can be found at http://www.mdpi.com/1422-0067/21/3/1068/s1. Figure S1. Bioinformatics analysis of StABI5. (A) Basic information about StABI5 on the Solanum tuberosum genome database website. (B) Phylogenetic tree analysis of ABI5 protein in Solanum tuberosum and other species. St: Solanum tuberosum, Sl: Solanum lycopersicum, Nt: Nicotiana tabacum, At: Arabidopsis thaliana, and Os: Oryza sativa. (C) Conserved domains analysis of StABI5. Figure S2. The phenotype of StABI5-OE transgenic potato lines planted in the field for one month. Figure S3. Validation of transcriptome data by qRT-PCR. Supplementary Table S1. Significantly enriched Go terms. Supplementary Table S2. Primers for quantitative real-time PCR in this study. 
Author Contributions: M.R., T.Z., and L.L. designed the experiments. T.Z. and L.L. performed the experiments. L.L. and L.F. analyzed the data. M.R., T.Z., and L.L. wrote the manuscript. All authors have read and agreed to the published version of the manuscript.

Funding: This work was supported by grants from the National Natural Science Foundation of China (No. 31672206 and 31972469), Chengdu Agricultural Science and Technology Center local financial special fund project (NASC2019TI13), and Central Public-interest Scientific Institution Basal Research Fund (No. Y2020XK04).

Conflicts of Interest: The authors declare no conflict of interest.

\section{References}

1. Lievens, L.; Pollier, J.; Goossens, A.; Beyaert, R.; Staal, J. Abscisic Acid as Pathogen Effector and Immune Regulator. Front. Plant Sci. 2017, 8, 587. [CrossRef]

2. Vishwakarma, K.; Upadhyay, N.; Kumar, N.; Yadav, G.; Singh, J.; Mishra, R.K.; Kumar, V.; Verma, R.; Upadhyay, R.G.; Pandey, M.; et al. Abscisic Acid Signaling and Abiotic Stress Tolerance in Plants: A Review on Current Knowledge and Future Prospects. Front. Plant Sci. 2017, 8, 161. [CrossRef] [PubMed]

3. Dong, T.; Park, Y.; Hwang, I. Abscisic acid: Biosynthesis, inactivation, homoeostasis and signalling. Essays Biochem. 2015, 58, 29-48. [PubMed]

4. Finkelstein, R.R. Mutations at two new Arabidopsis ABA response loci are similar to the abi3 mutations. Plant J. 1994, 5, 765-771. [CrossRef]

5. Finkelstein, R.R.; Lynch, T.J. The Arabidopsis abscisic acid response gene ABI5 encodes a basic leucine zipper transcription factor. Plant Cell 2000, 12, 599-609. [CrossRef]

6. Carles, C.; Bies-Etheve, N.; Aspart, L.; Léon-Kloosterziel, K.M.; Koornneef, M.; Echeverria, M.; Delseny, M. Regulation of Arabidopsis thaliana Em genes: Role of ABI5. Plant J. Cell Mol. Biol. 2002, 30, 373-383. [CrossRef]

7. Kanai, M.; Nishimura, M.; Hayashi, M. A peroxisomal ABC transporter promotes seed germination by inducing pectin degradation under the control of ABI5. Plant J. Cell Mol. Biol. 2010, 62, 936-947. [CrossRef]

8. Ju, L.; Jing, Y.; Shi, P.; Liu, J.; Chen, J.; Yan, J.; Chu, J.; Chen, K.M.; Sun, J. JAZ proteins modulate seed germination through interaction with ABI5 in bread wheat and Arabidopsis. New Phytol. 2019, 223, $246-260$. [CrossRef]

9. $\mathrm{Hu}, \mathrm{Y} . ; \mathrm{Yu}, \mathrm{D}$. BRASSINOSTEROID INSENSITIVE2 interacts with ABSCISIC ACID INSENSITIVE5 to mediate the antagonism of brassinosteroids to abscisic acid during seed germination in Arabidopsis. Plant Cell 2014, 26, 4394-4408. [CrossRef]

10. Zhao, X.; Dou, L.; Gong, Z.; Wang, X. BES1 hinders ABSCISIC ACID INSENSITIVE5 and promotes seed germination in Arabidopsis. New Phytol. 2018, 221, 908-918. [CrossRef]

11. Yang, X.; Bai, Y.; Shang, J.; Xin, R.; Tang, W. The antagonistic regulation of abscisic acid-inhibited root growth by brassinosteroids is partially mediated via direct suppression of ABSCISIC ACID INSENSITIVE 5 expression by BRASSINAZOLE RESISTANT 1. Plant Cell Environ. 2016, 39, 1994-2003. [CrossRef] [PubMed]

12. Hu, Y.; Han, X.; Yang, M.; Zhang, M.; Pan, J.; Yu, D. The Transcription Factor INDUCER OF CBF EXPRESSION1 Interacts with ABSCISIC ACID INSENSITIVE5 and DELLA Proteins to Fine-Tune Abscisic Acid Signaling during Seed Germination in Arabidopsis. Plant Cell 2019, 31, 1520-1538. [CrossRef] [PubMed]

13. Lim, S.; Park, J.; Lee, N.; Jeong, J.; Toh, S.; Watanabe, A.; Kim, J.; Kang, H.; Kim, D.H.; Kawakami, N.; et al. ABA-insensitive3, ABA-insensitive5, and DELLAs Interact to activate the expression of SOMNUS and other high-temperature-inducible genes in imbibed seeds in Arabidopsis. Plant Cell 2013, 25, 4863-4878. [CrossRef] [PubMed]

14. Kong, Y.; Chen, S.; Yang, Y.; An, C. ABA-insensitive (ABI) 4 and ABI5 synergistically regulate DGAT1 expression in Arabidopsis seedlings under stress. FEBS Lett. 2013, 587, 3076-3082. [CrossRef] [PubMed]

15. Sakuraba, Y.; Jeong, J.; Kang, M.Y.; Kim, J.; Paek, N.C.; Choi, G. Phytochrome-interacting transcription factors PIF4 and PIF5 induce leaf senescence in Arabidopsis. Nat. Commun. 2014, 5, 4636. [CrossRef] [PubMed]

16. Su, M.; Huang, G.; Zhang, Q.; Wang, X.; Li, C.; Tao, Y.; Zhang, S.; Lai, J.; Yang, C.; Wang, Y. The LEA protein, $\mathrm{ABR}$, is regulated by $\mathrm{ABI} 5$ and involved in dark-induced leaf senescence in Arabidopsis thaliana. Plant Sci. 2016, 247, 93-103. [CrossRef]

17. Signora, L.; De Smet, I.; Foyer, C.H.; Zhang, H. ABA plays a central role in mediating the regulatory effects of nitrate on root branching in Arabidopsis. Plant J. Cell Mol. Biol. 2001, 28, 655-662. [CrossRef] 
18. Bossi, F.; Cordoba, E.; Dupré, P.; Mendoza, M.S.; Román, C.S.; León, P. The Arabidopsis ABA-INSENSITIVE (ABI) 4 factor acts as a central transcription activator of the expression of its own gene, and for the induction of ABI5 and SBE2.2 genes during sugar signaling. Plant J. Cell Mol. Biol. 2009, 59, 359-374. [CrossRef]

19. Lopez-Molina, L.; Mongrand, S.; McLachlin, D.T.; Chait, B.T.; Chua, N.H. ABI5 acts downstream of ABI3 to execute an ABA-dependent growth arrest during germination. Plant J. Cell Mol. Biol. 2002, 32, 317-328. [CrossRef]

20. Xu, D.; Li, J.; Gangappa, S.N.; Hettiarachchi, C.; Lin, F.; Andersson, M.X.; Jiang, Y.; Deng, X.W.; Holm, M. Convergence of Light and ABA signaling on the ABI5 promoter. PLoS Genet. 2014, 10, e1004197. [CrossRef]

21. Chen, H.; Zhang, J.; Neff, M.M.; Hong, S.W.; Zhang, H.; Deng, X.W.; Xiong, L. Integration of light and abscisic acid signaling during seed germination and early seedling development. Proc. Natl. Acad. Sci. USA 2008, 105, 4495-4500. [CrossRef] [PubMed]

22. Seo, P.J.; Xiang, F.; Qiao, M.; Park, J.Y.; Lee, Y.N.; Kim, S.G.; Lee, Y.H.; Park, W.J.; Park, C.M. The MYB96 transcription factor mediates abscisic acid signaling during drought stress response in Arabidopsis. Plant Physiol. 2009, 151, 275-289. [CrossRef] [PubMed]

23. Kim, J.H.; Hyun, W.Y.; Nguyen, H.N.; Jeong, C.Y.; Xiong, L.; Hong, S.W.; Lee, H. AtMyb7, a subgroup 4 R2R3 $\mathrm{Myb}$, negatively regulates ABA-induced inhibition of seed germination by blocking the expression of the bZIP transcription factor ABI5. Plant Cell Environ. 2015, 38, 559-571. [CrossRef] [PubMed]

24. Liu, Z.Q.; Yan, L.; Wu, Z.; Mei, C.; Lu, K.; Yu, Y.T.; Liang, S.; Zhang, X.F.; Wang, X.F.; Zhang, D.P. Cooperation of three WRKY-domain transcription factors WRKY18, WRKY40, and WRKY60 in repressing two ABA-responsive genes ABI4 and ABI5 in Arabidopsis. J. Exp. Bot. 2012, 63, 6371-6392. [CrossRef]

25. Lynch, T.; Erickson, B.J.; Finkelstein, R.R. Direct interactions of ABA-insensitive(ABI)-clade protein phosphatase(PP)2Cs with calcium-dependent protein kinases and ABA response element-binding bZIPs may contribute to turning off ABA response. Plant Mol. Biol. 2012, 80, 647-658. [CrossRef]

26. Lyzenga, W.J.; Liu, H.; Schofield, A.; Muise-Hennessey, A.; Stone, S.L. Arabidopsis CIPK26 interacts with KEG, components of the ABA signalling network and is degraded by the ubiquitin-proteasome system. J. Exp. Bot. 2013, 64, 2779-2791. [CrossRef]

27. Fujii, H.; Zhu, J.K. An Arabidopsis mutant deficient in all three abscisic acid-activated protein kinases reveals critical roles in plant growth, reproduction and stress. Proc. Natl. Acad. Sci. USA 2009, 106, 8380-8385. [CrossRef]

28. Hu, R.; Zhu, Y.; Shen, G.; Zhang, H. TAP46 plays a positive role in the ABSCISIC ACID INSENSITIVE5regulated gene expression in Arabidopsis. Plant Physiol. 2014, 164, 721-734. [CrossRef]

29. Dai, M.; Xue, Q.; Mccray, T.; Margavage, K.; Chen, F.; Lee, J.H.; Nezames, C.D.; Guo, L.; Terzaghi, W.; Wan, J.; et al. The PP6 Phosphatase Regulates ABI5 Phosphorylation and Abscisic Acid Signaling in Arabidopsis. Plant Cell 2013, 25, 517-534. [CrossRef]

30. Lee, J.H.; Yoon, H.J.; Terzaghi, W.; Martinez, C.; Dai, M.; Li, J.; Byun, M.O.; Deng, X.W. DWA1 and DWA2, two Arabidopsis DWD protein components of CUL4-based E3 ligases, act together as negative regulators in ABA signal transduction. Plant Cell 2010, 22, 1716-1732. [CrossRef]

31. Stone, S.L.; Williams, L.A.; Farmer, L.M.; Vierstra, R.D.; Callis, J. KEEP ON GOING, a RING E3 ligase essential for Arabidopsis growth and development, is involved in abscisic acid signaling. Plant Cell 2006, 18, 3415-3428. [CrossRef] [PubMed]

32. Liu, H.; Stone, S.L. Regulation of ABI5 turnover by reversible post-translational modifications. Plant Signal. Behav. 2014, 9, e27577. [CrossRef] [PubMed]

33. Skubacz, A.; Daszkowska-Golec, A.; Szarejko, I. The Role and Regulation of ABI5 (ABA-Insensitive 5) in Plant Development, Abiotic Stress Responses and Phytohormone Crosstalk. Front. Plant Sci. 2016, 7, 1884. [CrossRef] [PubMed]

34. So-Yon, P.; Yu, J.W.; Park, J.S.; Li, J.; Yoo, S.C.; Lee, N.Y.; Lee, S.K.; Jeong, S.W.; Seo, H.S.; Koh, H.J.; et al. The senescence-induced staygreen protein regulates chlorophyll degradation. Plant Cell 2007, 19, 1649-1664.

35. Kusaba, M.; Ito, H.; Morita, R.; Iida, S.; Sato, Y.; Fujimoto, M.; Kawasaki, S.; Tanaka, R.; Hirochika, H.; Nishimura, M.; et al. Rice NON-YELLOW COLORING1 is involved in light-harvesting complex II and grana degradation during leaf senescence. Plant Cell 2007, 19, 1362-1375. [CrossRef]

36. Leutwiler, L.S.; Meyerowitz, E.M.; Tobin, E.M. Structure and expression of three light-harvesting chlorophyll a/b-binding protein genes in Arabidopsis thaliana. Nucleic Acids Res. 1986, 14, 4051-4064. [CrossRef]

37. Wilson, R.H.; Hayer-Hartl, M. Complex Chaperone Dependence of Rubisco Biogenesis. Biochemistry 2018, 57, 3210-3216. [CrossRef] 
38. Kamranfar, I.; Xue, G.P.; Tohge, T.; Sedaghatmehr, M.; Fernie, A.R.; Balazadeh, S.; Mueller-Roeber, B. Transcription factor RD26 is a key regulator of metabolic reprogramming during dark-induced senescence. New Phytol. 2018, 218, 1543-1557. [CrossRef]

39. Wang, S.; Blumwald, E. Stress-induced chloroplast degradation in Arabidopsis is regulated via a process independent of autophagy and senescence-associated vacuoles. Plant Cell 2014, 26, 4875-4888. [CrossRef]

40. Waters, M.T.; Langdale, J.A. The making of a chloroplast. EMBO J. 2009, 28, 2861-2873. [CrossRef]

41. Yu, F.; Wu, Y.; Xie, Q. Precise protein post-translational modifications modulate ABI5 activity. Trends Plant Sci. 2015, 20, 569-575. [CrossRef] [PubMed]

42. Xiong, F.; Zhang, R.; Meng, Z.; Deng, K.; Que, Y.; Zhuo, F.; Feng, L.; Guo, S.; Datla, R.; Ren, M. Brassinosteriod Insensitive 2 (BIN2) acts as a downstream effector of the Target of Rapamycin (TOR) signaling pathway to regulate photoautotrophic growth in Arabidopsis. New Phytol. 2017, 213, 233-249. [CrossRef] [PubMed]

43. Chen, K.; Li, G.J.; Bressan, R.A.; Song, C.P.; Zhu, J.K.; Zhao, Y. Abscisic acid dynamics, signaling and functions in plants. J. Integr. Plant Biol. 2019. [CrossRef] [PubMed]

44. Kumar, M.; Kesawat, M.S.; Ali, A.; Lee, S.C.; Gill, S.S.; Kim, H.U. Integration of Abscisic Acid Signaling with Other Signaling Pathways in Plant Stress Responses and Development. Plants 2019, 8, 592. [CrossRef]

45. Yuan, T.T.; Xu, H.H.; Zhang, K.X.; Guo, T.T.; Lu, Y.T. Glucose inhibits root meristem growth via ABA INSENSITIVE 5, which represses PIN1 accumulation and auxin activity in Arabidopsis. Plant Cell Environ. 2014, 37, 1338-1350. [CrossRef]

46. Asad, M.A.U.; Zakari, S.A.; Zhao, Q.; Zhou, L.; Ye, Y.; Cheng, F. Abiotic Stresses Intervene with ABA Signaling to Induce Destructive Metabolic Pathways Leading to Death: Premature Leaf Senescence in Plants. Int. J. Mol. Sci. 2019, 20, 256. [CrossRef]

47. Imamura, S.; Nomura, Y.; Takemura, T.; Pancha, I.; Taki, K.; Toguchi, K.; Tozawa, Y.; Tanaka, K. The checkpoint kinase TOR (target of rapamycin) regulates expression of a nuclear-encoded chloroplast RelA-SpoT homolog (RSH) and modulates chloroplast ribosomal RNA synthesis in a unicellular red alga. Plant J. Cell Mol. Biol. 2018, 94, 327-339. [CrossRef]

48. Rojas-Pierce, M.; Whippo, C.W.; Davis, P.A.; Hangarter, R.P.; Springer, P.S. PLASTID MOVEMENT IMPAIRED1 mediates ABA sensitivity during germination and implicates ABA in light-mediated Chloroplast movements. Plant Physiol. Biochem. 2014, 83, 185-193. [CrossRef]

49. Jarvis, P.; Lopez-Juez, E. Biogenesis and homeostasis of chloroplasts and other plastids. Nat. Rev. Mol. Cell Biol. 2013, 14, 787-802. [CrossRef]

50. Li, L.; Song, Y.; Wang, K.; Dong, P.; Zhang, X.; Li, F.; Li, Z.; Ren, M. TOR-inhibitor insensitive-1 (TRIN1) regulates cotyledons greening in Arabidopsis. Front. Plant Sci. 2015, 6, 861. [CrossRef]

51. Leon, P.; Gregorio, J.; Cordoba, E. ABI4 and its role in chloroplast retrograde communication. Front. Plant Sci. 2012, 3, 304. [CrossRef] [PubMed]

52. Acevedo-Hernandez, G.J.; Leon, P.; Herrera-Estrella, L.R. Sugar and ABA responsiveness of a minimal RBCS light-responsive unit is mediated by direct binding of ABI4. Plant J. Cell. Mol. Biol. 2005, 43, 506-519. [CrossRef] [PubMed]

53. Wang, P.; Grimm, B. Organization of chlorophyll biosynthesis and insertion of chlorophyll into the chlorophyll-binding proteins in chloroplasts. Photosynth. Res. 2015, 126, 189-202. [CrossRef] [PubMed]

54. Ozawa, S.I.; Bald, T.; Onishi, T.; Xue, H.; Matsumura, T.; Kubo, R.; Takahashi, H.; Hippler, M.; Takahashi, Y. Configuration of Ten Light-Harvesting Chlorophyll a/b Complex I Subunits in Chlamydomonas reinhardtii Photosystem, I. Plant Physiol. 2018, 178, 583-595. [CrossRef]

55. Weaver, L.M.; Amasino, R.M. Senescence is induced in individually darkened Arabidopsis leaves, but inhibited in whole darkened plants. Plant Physiol. 2001, 127, 876-886. [CrossRef]

56. Kim, D.; Langmead, B.; Salzberg, S.L. HISAT: A fast spliced aligner with low memory requirements. Nat. Methods 2015, 12, 357-360. [CrossRef]

57. Love, M.I.; Huber, W.; Anders, S. Moderated estimation of fold change and dispersion for RNA-seq data with DESeq2. Genome Biol. 2014, 15, 550. [CrossRef]

(C) 2020 by the authors. Licensee MDPI, Basel, Switzerland. This article is an open access article distributed under the terms and conditions of the Creative Commons Attribution (CC BY) license (http://creativecommons.org/licenses/by/4.0/). 